\title{
Clinical utility of antimicrobial susceptibility measurement plate covering formulated concentrations of various ophthalmic antimicrobial drugs
}

\author{
This article was published in the following Dove Press journal: \\ Clinical Ophthalmology \\ 9 November 2016 \\ Number of times this article has been viewed
}

\author{
Norihiko Tou' \\ Ryohei Nejima ${ }^{2}$ \\ Yoshifumi Ikeda ${ }^{3}$ \\ Yuichi Hori ${ }^{4}$ \\ Kaoru Araki-Sasaki ${ }^{5}$ \\ Kazunori Miyata ${ }^{2}$ \\ Yoshitsugu Inoue ${ }^{3}$ \\ Akihiko Tawara' \\ 'Department of Ophthalmology, \\ University of Occupational and \\ Environmental Health, Fukuoka, \\ ${ }^{2}$ Miyata Eye Hospital, Miyazaki, \\ ${ }^{3}$ Division of Ophthalmology and Visual \\ Science, Faculty of Medicine, Tottori \\ University, Tottori, ${ }^{4}$ Department of \\ Ophthalmology, Toho University \\ Sakura Medical Center, Chiba, ${ }^{5}$ deta \\ Eye Hospital, Kumamoto, Japan
}

Purpose: The purpose of this study was to evaluate the clinical utility of SG17, an ophthalmic antimicrobial susceptibility measurement plate.

Design: This was a multicenter, retrospective, observational study.

Patients and methods: Using clinical isolates from patients with ocular infections, drug susceptibility testing using the Clinical Laboratory and Standards Institute standards was routinely conducted at five facilities. The minimum inhibitory concentrations of the isolated strains were determined using SG17 at the Research Foundation for Microbial Diseases of Osaka University. The records of antimicrobial drugs used and the therapeutic course were evaluated for all cases. The susceptibility results from SG17 and routine methods used at each facility were compared.

Results: A total of 112 bacterial strains were isolated from 92 patients. Of these cases, keratitis was the most common (52.2\%), followed by conjunctivitis (21.7\%) and others (26.1\%). Principal signs and symptoms resolved in all patients, indicating that therapeutic effects had been achieved. With SG17, drug susceptibility was determined in $98.9 \%$ of isolates compared with $30.4 \%$ of isolates determined using conventional methods. By adapting the Clinical Laboratory and Standards Institute standards to SG17 results, we found that $91.3 \%$ of patients were susceptible and $7.6 \%$ were resistant. In five patients, drugs with a resistant evaluation were initially administered with no effect, and the patients were then switched to drugs with a susceptible evaluation with final resolution, indicating agreement of clinical results with SG17.

Conclusion: SG17 can be used to determine drug susceptibility to antimicrobial agents currently used in ophthalmic practice. SG17 is useful for selecting antimicrobial drugs.

Keywords: ophthalmic antimicrobial agent, drug susceptibility, minimum inhibitory concentration, Clinical Laboratory and Standards Institute

\section{Introduction}

Evaluating the drug susceptibility of causative microorganisms is important for selecting the most effective antimicrobial agents against ocular infections. ${ }^{1-5}$ Susceptibility testing is determined by the minimum inhibitory concentration (MIC), although numerous facilities use the standards issued by the Clinical Laboratory and Standards Institute (CLSI) in actual practice as the basis for the selection of therapeutics. ${ }^{6}$ Several issues exist when applying the CLSI standards to ophthalmic antimicrobial agents. First, the standards were created based on the pharmacodynamics of antimicrobial drugs administered systemically at the dose and administration method approved in
Correspondence: Norihiko Tou Department of Ophthalmology, University of Occupational and Environmental Health, Japan, Iseigaoka I-I,

Yahatanishi-ku, Kitakyushu-shi,

Fukuoka 807-8555, Japan

$\mathrm{Tel}+8|9369| 726 \mid$

Fax +8I 936033657

Email n-toh@med.uoeh-u.ac.jp 
the United States of America. Owing to changes in the administration route and concentration, actual clinical results may differ. Second, topical ophthalmic antimicrobial drugs can achieve a much higher concentration on the ocular surface than those administered systemically, even when considering dilution through tears and the immediate excretion by blinking after instillation. Third, the antimicrobial drugs used for susceptibility testing are primarily designed for systemic administration, and those available only in ophthalmic solutions have not been evaluated. Previous studies have shown a correlation between the MIC value and clinical outcomes in patients with corneal infections. ${ }^{7-9}$ Therefore, it is essential to determine the MIC of antimicrobial ophthalmic solutions used in clinical practice to treat ocular infections.

In this study, we describe a drug susceptibility testing plate, SG17 (Eiken Chemical, Tokyo, Japan), developed as an MIC measurement panel. This plate measures the MIC of a drug from low to high concentrations, covering many types and formulated concentrations of antimicrobial drugs (including ophthalmic preparations) commonly used in Japan to treat ocular infections. The clinical utility of this plate was tested at multiple facilities.

\section{Patients and methods}

This multicenter, retrospective, observational study was approved by the Ethics Committee of Occupational and Environmental Health, Tottori University, Ideta Eye Hospital and Miyata Eye Hospital, and based on the decision and approval of each committee, the participation of this study was announced to patients by bulletin board, instead of by obtaining individual consent of each patient. All procedures were in accordance with the tenets of the Declaration of Helsinki.

Ninety-two patients diagnosed with ocular infections presented at five facilities in Japan from November 2009 to August 2011, and 112 microbial strains were isolated. Subjects were excluded if concomitant topical or systemic steroids or nonsteroidal anti-inflammatory drugs were newly prescribed together with antimicrobial drugs for treatment.

The hospital laboratory of each facility performed the microbial culture, identification, and susceptibility testing using routine methods. Two laboratories determined the susceptibility using the CLSI broth dilution method ${ }^{10,11}$ with commercially available susceptibility plates and the remaining three laboratories determined the susceptibility using the CLSI disk diffusion method; ${ }^{12}$ strains were evaluated as susceptible (S), intermediate (I), or resistant (R). At the same time, the isolates were frozen in cryogenic vials and stored. Clinical records, including background diseases, main symptoms, main clinical signs, antimicrobial drugs used, and therapeutic course, were collected. Treatment outcomes were evaluated by changes in the main symptoms and clinical signs, and complete resolution was considered cured.

The frozen strains were sent to the Research Foundation for Microbial Diseases of Osaka University, one of the main research laboratories in Japan, and MICs were determined for the 12 antibiotics using SG17 based on the CLSI broth dilution method. ${ }^{10,11}$ The details of MIC measurement are as follows. The isolates were cultured overnight on a nonselective agar medium and suspended in sterile saline with the turbidity adjusted to level 1 of the McFarland scale. The bacterial suspension for inoculation was adjusted with $0.25 \mathrm{~mL}$ of the saline suspension and $12 \mathrm{~mL}$ of Mueller Hinton medium. From this solution, $0.1 \mathrm{~mL}$ was added to each well to obtain a final concentration of $5 \times 10^{4}$ colony forming units/well. After inoculation, the plates were incubated at $35^{\circ} \mathrm{C} \pm 2{ }^{\circ} \mathrm{C}$ for $16-24$ hours. The medium and culture conditions varied according to the bacterial species. The MIC was the lowest concentration of antimicrobial agent that completely inhibited the growth of the organism in the wells, as detected by the unaided eye. Figure 1 shows the types and concentrations of antimicrobial agents placed in SG17.

Information obtained from the susceptibility results by SG17, S/I/R determination at the facilities, clinical signs, and treatment outcomes were compared, and the clinical utility of SG17 was evaluated. The CLSI standards were compared to the MIC as determined by SG17 and evaluated for determination of $\mathrm{S} / \mathrm{I} / \mathrm{R}$ status as well. There were several cases in which CLSI standards did not exist. For example, to evaluate Corynebacterium spp., we substituted the evaluation for Staphylococcus spp.; to evaluate ofloxacin results, twice the breaking point of levofloxacin was substituted; and to evaluate cefmenoxime, the breaking point of ceftazidime (another cephalosporin drug of the same generation) was substituted.

\section{Results}

\section{Patient characteristics}

The mean patient age (mean \pm standard deviation) was $62.6 \pm 22.7$ years (range, $0-92$ years). There were 54 females (58.7\%) and 38 males (41.3\%); 48 right eyes (52.2\%) and 44 left eyes $(47.8 \%)$ were included. Table 1 shows the characteristics of the ocular infections. All patients eventually had complete resolution. The mean time required for resolution was $25.7 \pm 28.5$ days (range, 3-154 days). In 112 strains obtained from patients, coagulase-negative Staphylococci were the most frequent (35 isolates; $31.3 \%$ ), followed by Corynebacterium spp. (28 isolates; 25\%), methicillin-sensitive Staphylococcus aureus (nine isolates; 


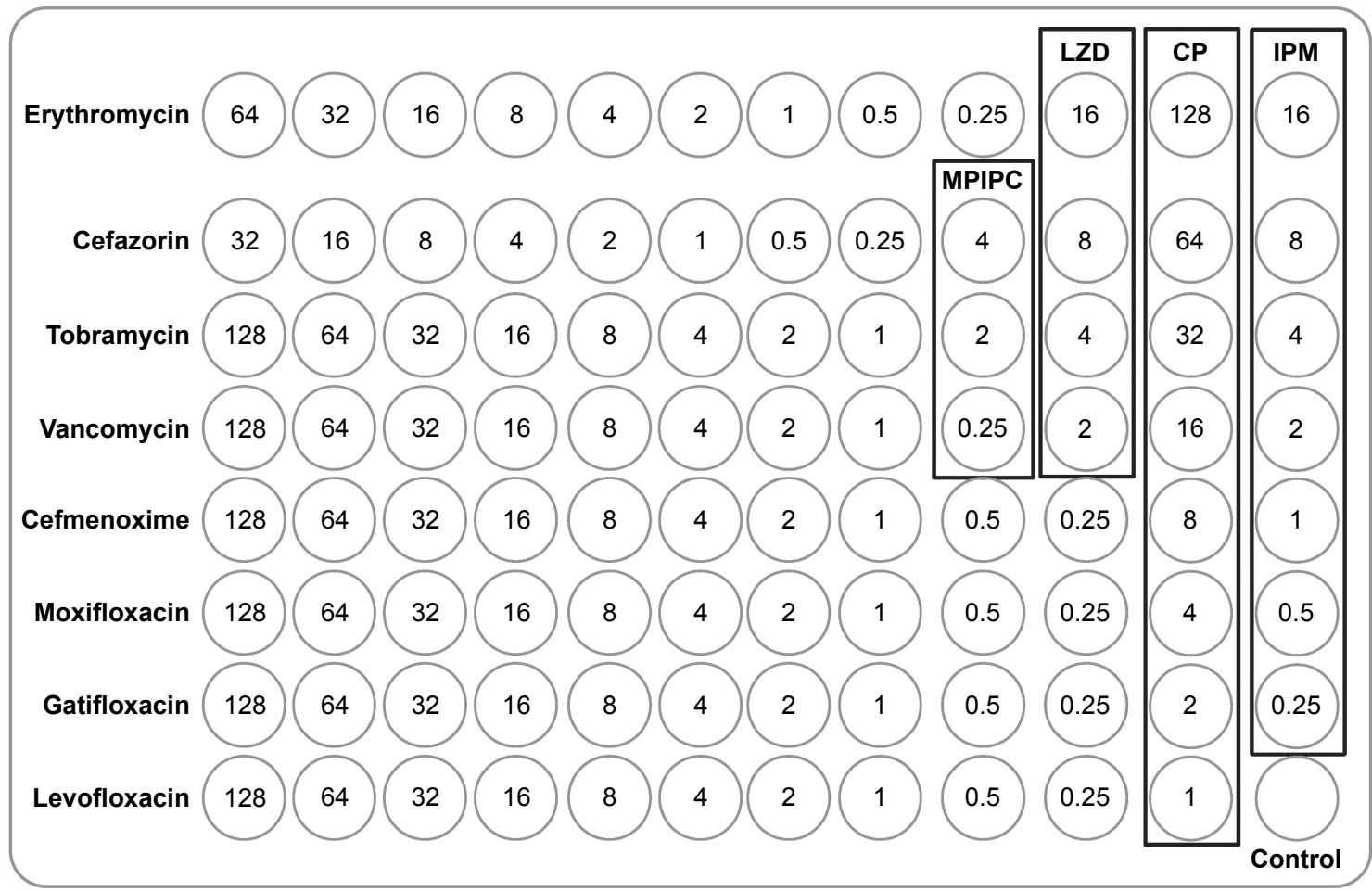

Figure I Arrangement of antimicrobial agents on SG 17, a susceptibility testing dry plate. Numbers in circles are concentration of the drugs ( $\mu g / \mathrm{mL})$. Abbreviations: MPIPC, oxacillin; LZD, linezolid; CP, chloramphenicol; IPM, imipenem.

8\%), methicillin-resistant S. aureus (eight isolates; 7.1\%), Pseudomonas aeruginosa (four isolates; $3.6 \%$ ), and Serratia marcescens (three isolates; $2.7 \%$ ). Fourteen isolates (12.5\%) of other gram-positive strains and eleven isolates $(9.8 \%)$ of gram-negative strains were detected.

Table I Characteristics of ocular infections

\begin{tabular}{ll}
\hline Characteristics (N=92) & $\mathbf{n}(\%)$ \\
\hline Disease & \\
Keratitis & $48(52.2)$ \\
Conjunctivitis & $20(21.7)$ \\
Dacryocystitis & $5(5.4)$ \\
Endophthalmitis & $4(4.3)$ \\
Blepharoconjunctivitis & $6(6.5)$ \\
Others & $9(9.8)$ \\
Antibiotics used & \\
Cefmenoxime & \\
Moxifloxacin & $55(59.8)$ \\
Levofloxacin & $43(46.7)$ \\
Gatifloxacin & $12(13)$ \\
Ofloxacin & $1 \mathrm{I}(\mathrm{I} 2)$ \\
Tosufloxacin & $2(2.2)$ \\
Ofloxacin (ointment) & $\mathrm{I}(\mathrm{I} . \mathrm{I})$ \\
Vancomycin & $42(45.7)$ \\
Chloramphenicol & $6(6.5)$ \\
Tobramycin & $6(6.5)$ \\
Erythromycin & $6(6.5)$ \\
Erythromycin (ointment) & $3(3.3)$ \\
\hline Note: & $\mathrm{I}(\mathrm{I} . \mathrm{I})$ \\
\hline
\end{tabular}

Note: averlapped cases.

\section{Drug susceptibility}

The MICs for $90 \%$ of isolates $\left(\mathrm{MIC}_{90}\right)$, as determined by SG17, are shown in Table 2. SG17 was able to measure the MICs and evaluate the S/I/R statuses of all the antimicrobial agents. In contrast, MIC measurements and S/I/R evaluations of antimicrobial agents that could be determined using conventional methods were as follows: cefmenoxime (six isolates; 5.4\%), tobramycin (seven isolates; 6.3\%), chloramphenicol (19 isolates; $17 \%$ ), erythromycin (47 isolates; 42\%), levofloxacin (111 isolates; 99.1\%), gatifloxacin (six isolates; 5.4\%), and moxifloxacin (six isolates; $5.4 \%$ ). Of the 111 isolates applied for measurement of the MIC of levofloxacin, 60 isolates (54.1\%) were $\mathrm{S}$, two isolates (1.8\%) were I, and 49 isolates (44.1\%) were R. The one isolate that was not measured for levofloxacin susceptibility was a nonfermenting gram-negative rod, and the MIC as determined by $\mathrm{SG} 17$ was $\leq 0.25 \mu \mathrm{g} / \mathrm{mL}$.

The MIC results of levofloxacin, gatifloxacin, and moxifloxacin of 49 isolates with $R$ evaluation of levofloxacin by routine methods at the various facilities ranged from $\leq 0.25 \mu \mathrm{g} / \mathrm{mL}$ to $>128 \mu \mathrm{g} / \mathrm{mL}$, as determined by SG17 (Figure 2).

\section{Clinical utility of SGI7}

MICs measured by SG17 were evaluated by the CLSI standards and compared with the $\mathrm{S} / \mathrm{I} / \mathrm{R}$ evaluation at the 
Table 2 Ninety percent MIC determined by SGI7

\begin{tabular}{|c|c|c|c|c|c|c|c|c|c|c|c|}
\hline \multirow[t]{2}{*}{ Isolated strains (n) } & \multicolumn{11}{|c|}{ MIC for $90 \%$ of isolates $(\mu \mathrm{g} / \mathrm{mL})$} \\
\hline & CMX & ТОВ & CP & EM & LVFX & GFLX & MFLX & CAZ & VCM & LZD & IPM \\
\hline \multicolumn{12}{|l|}{ Gram-positive bacteria (94) } \\
\hline Coagulase-negative Staphylococci (35) & 8 & 64 & 64 & $>64$ & 32 & 8 & 8 & 32 & 2 & $\leq 2$ & I \\
\hline Corynebacterium spp. (28) & 1 & 64 & 32 & 64 & $>128$ & 64 & 128 & 16 & $\leq 1$ & 4 & 2 \\
\hline S. aureus, including MRSA (17) & $>128$ & 128 & 16 & $>64$ & $>128$ & 128 & 64 & $>128$ & 2 & 4 & $>16$ \\
\hline Other gram-positive bacteria (14) & 16 & 64 & 4 & $>64$ & 2 & I & 0.5 & 16 & $\leq 1$ & $\leq 2$ & 0.5 \\
\hline Gram-negative bacteria $(18)$ & $>128$ & 32 & 64 & $>64$ & 4 & 4 & 2 & $>128$ & $>128$ & $>16$ & $>16$ \\
\hline Total $(|| 2)$ & 16 & 64 & 64 & $>64$ & $>128$ & 32 & 64 & 16 & 128 & $>16$ & 16 \\
\hline
\end{tabular}

Abbreviations: MIC, minimum inhibitory concentration; CMX, cefmenoxime; TOB, tobramycin; CP, chloramphenicol; EM, erythromycin; LVFX, levofloxacin; GFLX, gatifloxacin; MFLX, moxifloxacin; CAZ, cefazorin; VCM, vancomycin; LZD, linezolid; IPM, imipenem; S. aureus, Staphylococcus aureus; MRSA, methicillin-resistant S. aureus.

various laboratories. The types of medication(s) used for the final treatment and the treatment outcomes were also evaluated.

Of 92 patients, the susceptibility of drugs used for treatment was able to be determined in 91 patients $(98.9 \%)$ by SG17 and in 28 patients (30.4\%) by facility tests. SG17 was clinically more useful. In only one patient $(1.1 \%)$, tosufloxacin was used, and SG17 could not evaluate the susceptibility of the isolate to this drug. Table 3 shows representative cases with the clinical course explainable only by SG17 for which the treatment was effective.

SG17 led to an S evaluation for drugs used in 84 patients $(91.3 \%)$ in which a therapeutic effect was finally achieved. Among these 84 patients, 13 patients (15.5\%) did not achieve a therapeutic effect with the first therapy and were then switched to the second therapy. Among these 13 patients, medications with an R evaluation by SG17 were

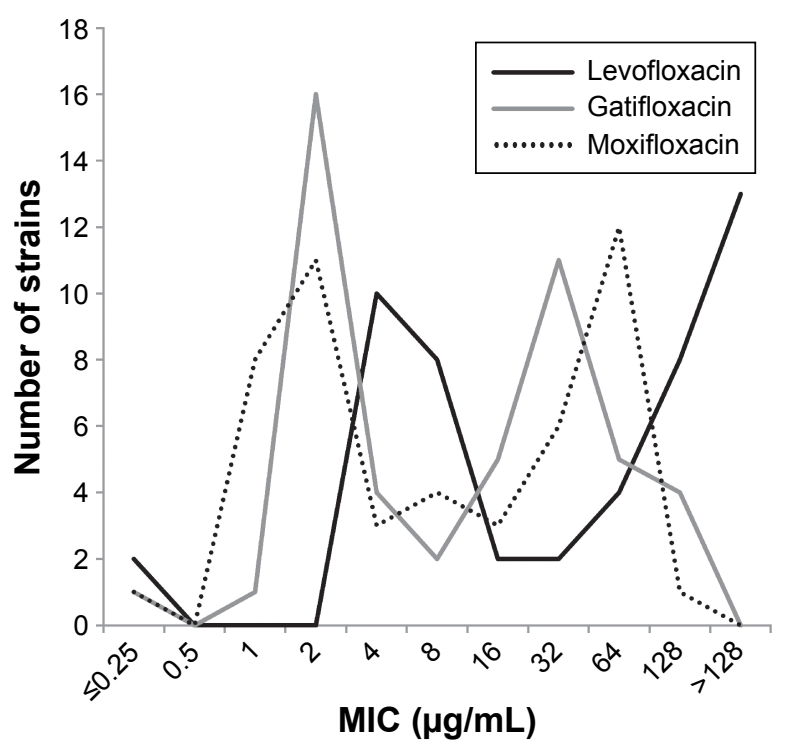

Figure 2 MIC distribution of fluoroquinolones by SG 17 in 49 isolates evaluated R to levofloxacin by usual methods at each facility.

Abbreviations: MIC, minimum inhibitory concentration; R, resistant. administered in five patients; however, the drugs were then switched to those with an S evaluation by SG17 due to lack of effect. The representative cases are described below (cases 1 and 2 in Table 4).

\section{Case I}

Case 1 was an 89-year-old woman who presented after bilateral cataract surgery, complicated by Descemet's membrane detachment, with an unstable cornea. The patient had received twice daily instillations of $0.1 \%$ betamethasone and $0.5 \%$ levofloxacin ophthalmic solutions and visited the clinic with a complaint of discharge from the left eye. The discharge was cultured, and eye instillation of $0.5 \%$ levofloxacin was switched to $0.5 \%$ cefmenoxime instillation four times daily, resulting in the disappearance of discharge. Corynebacterium sp. was isolated from culture. The test at the facility showed that the isolate was R to levofloxacin and had unknown susceptibility to cefmenoxime; however, the determination by SG17 was S to cefmenoxime at $\leq 0.25 \mu \mathrm{g} / \mathrm{mL}$ and $\mathrm{R}$ to levofloxacin at $128 \mu \mathrm{g} / \mathrm{mL}$, in agreement with the clinical course.

\section{Case 2}

Case 2 was a 70-year-old woman. During her hospitalization for acute promyelocytic leukemia, she complained of pain, eyelid swelling, and discharge from her left eye for 3 days and was referred to the ophthalmology department on September 29,2010 . There was bilateral prominent conjunctival hyperemia with ample discharge, particularly evident in the left eye. The patient was diagnosed with bacterial conjunctivitis. The conjunctival swab was cultured, and the patient was prescribed $0.3 \%$ gatifloxacin instillation (four times daily) and $0.5 \%$ cefmenoxime (four times daily). Ten days later, after no improvement in symptoms, therapy was switched to $0.5 \%$ vancomycin instillation, and symptoms readily improved. The isolated methicillin-resistant $S$. aureus was determined 
Table 3 Representative cases in which SGI7 was clinically more useful than facility testing

\begin{tabular}{|c|c|c|c|c|c|}
\hline $\begin{array}{l}\text { Case } \\
\text { number }\end{array}$ & Disease & Species & Drug used $^{a}$ & $\begin{array}{l}\text { Susceptibility } \\
\text { at each institute }\end{array}$ & $\begin{array}{l}\text { MIC measured } \\
\text { by SGI7 (S, I, R) }\end{array}$ \\
\hline \multirow[t]{2}{*}{ I } & Conjunctivitis & S. epidermidis & MFLX & ND & $2(\mathrm{R})$ \\
\hline & & & CMX & ND & $0.5\left(S^{b}\right)$ \\
\hline 2 & Conjunctivitis & Corynebacterium sp. & CMX & ND & $\leq 0.25\left(\mathrm{~S}^{\mathrm{b}}\right)$ \\
\hline \multirow[t]{2}{*}{3} & Keratitis & S. epidermidis & EM & $\mathrm{R}$ & $32(\mathrm{R})$ \\
\hline & & & CMX & ND & $\mathrm{I}\left(\mathrm{S}^{\mathrm{b}}\right)$ \\
\hline
\end{tabular}

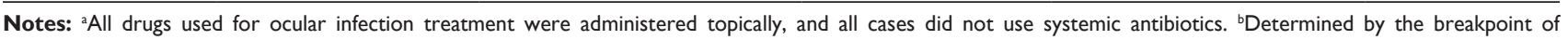
ceftazidime.

Abbreviations: MIC, minimum inhibitory concentration; S, susceptible; I, intermediate; R, resistant; S. epidermidis, Staphylococcus epidermidis; MFLX, moxifloxacin; CMX, cefmenoxime; ND, not determined; EM, erythromycin.

to be $\mathrm{R}$ to gatifloxacin at $32 \mu \mathrm{g} / \mathrm{mL}, \mathrm{R}$ to cefmenoxime at $>128 \mu \mathrm{g} / \mathrm{mL}$, and $\mathrm{S}$ to vancomycin at $\leq 1 \mu \mathrm{g} / \mathrm{mL}$ by SG17; this was in agreement with the clinical course that improved with the change in therapy.

Table 4 shows cases including these two cases (cases 1 and 2) with a clinical course explainable by SG17 in which the first treatment failed and the subsequent treatment was effective. Among 13 patients who switched medications, in seven patients, medications with an S evaluation by SG-17 were administered from the beginning, but were switched to other medications with an S evaluation with lower MICs by SG17 during treatment.

SG17 led to an R evaluation in seven patients (7.6\%). Despite this $\mathrm{R}$ evaluation, therapeutic effects were achieved in these seven patients.

\section{Discussion}

In this study, we designed and evaluated SG17, a drug susceptibility testing plate, for determination of a wide range of MICs for ophthalmic antimicrobial agents commonly used in Japan. We previously introduced the SG07 plate for the same purpose ${ }^{13}$ however, this plate did not include erythromycin and other drugs had concentrations that were too high for clinical purposes. With SG17, we were able to overcome these issues. The results of our current analyses showed that SG17 could be used to successfully evaluate the MICs of clinical isolates from patients with ocular infections from five facilities in Japan. Together with evaluation of the efficacy of antibiotics in these patients, our results suggested that SG17 has clinical applications in the determination of MICs for ophthalmic antimicrobials.

Most facilities use the CLSI standards to report the results of susceptibility testing to clinicians. However, the susceptibilities of antimicrobial drugs routinely used in ophthalmic practices have not been determined, and the currently used $\mathrm{S} / \mathrm{I} / \mathrm{R}$ evaluations do not reflect the concentrations of drugs on ocular tissues after the administration of eye drops. As such, the CLSI standards do not achieve the goal of selecting clinically effective ocular antimicrobial agents for causative microorganisms.

In this study, none of the laboratories were able to determine the susceptibility of antimicrobial agents for $>50 \%$ of the drugs used for treatment, with the sole

Table 4 Cases in which SGI7 could explain clinical results before and after drug switching

\begin{tabular}{|c|c|c|c|c|c|c|}
\hline \multirow{2}{*}{$\begin{array}{l}\text { Case } \\
\text { number }\end{array}$} & \multirow[t]{2}{*}{ Disease } & \multirow[t]{2}{*}{ Species } & \multicolumn{2}{|c|}{ Before switch } & \multicolumn{2}{|c|}{ After switch } \\
\hline & & & $\begin{array}{l}\text { Drug } \\
\text { used }^{a}\end{array}$ & $\begin{array}{l}\text { MIC measured } \\
\text { by SG I } 7(S, I, R)\end{array}$ & $\begin{array}{l}\text { Drug } \\
\text { used }^{a}\end{array}$ & $\begin{array}{l}\text { MIC measured } \\
\text { by SG I } 7(S, I, R)\end{array}$ \\
\hline 1 & Conjunctivitis & Corynebacterium sp. & LVFX & $128(\mathrm{R})$ & CMX & $\leq 0.25(\mathrm{~S})$ \\
\hline \multirow[t]{2}{*}{2} & Conjunctivitis & MRSA & GFLX & $32(\mathrm{R})$ & VCM & $\leq \mathrm{I}(\mathrm{S})$ \\
\hline & & & CMX & $>128\left(R^{b}\right)$ & & \\
\hline \multirow[t]{2}{*}{3} & Conjunctivitis & S. epidermidis & MFLX & $2(\mathrm{R})$ & MFLX & $2(\mathrm{R})$ \\
\hline & & & & & CMX & $0.5\left(\mathrm{~S}^{\mathrm{b}}\right)$ \\
\hline \multirow[t]{2}{*}{4} & Dacryocystitis & S. epidermidis & LVFX & $4(\mathrm{R})$ & $\mathrm{CP}$ & $64(\mathrm{R})$ \\
\hline & & & & & VCM & $\leq \mathrm{I}(\mathrm{S})$ \\
\hline \multirow[t]{2}{*}{5} & Conjunctivitis & Corynebacterium sp. & LVFX & $128(R)$ & CMX & $0.25\left(\mathrm{~S}^{\mathrm{b}}\right)$ \\
\hline & & MRSA & LVFX & $0.5(\mathrm{~S})$ & CMX & $4\left(S^{b}\right)$ \\
\hline
\end{tabular}

Notes: all drugs used for ocular infection treatment were administered topically, and all cases did not use systemic antibiotics. ${ }^{b}$ Determined by the breakpoint of ceftazidime.

Abbreviations: MIC, minimum inhibitory concentration; S, susceptible; I, intermediate; R, resistant; LVFX, levofloxacin; CMX, cefmenoxime; MRSA, methicillinresistant S. aureus; GFLX, gatifloxacin; VCM, vancomycin; S. epidermidis, Staphylococcus epidermidis; MFLX, moxifloxacin; CP, chloramphenicol. 
exception of levofloxacin. A primary reason for this is that many facilities use testing plates designed for systemic, not ophthalmic, medical treatment.

We also included gatifloxacin and moxifloxacin because testing at each facility typically only included a single fluoroquinolone levofloxacin. However, the MIC distributions of fluoroquinolones toward strains with an $\mathrm{R}$ determination to levofloxacin by routine methods ranged from low to high concentrations, as determined using SG17. Thus, these results indicated that the susceptibility of levofloxacin could not be substituted with that of other fluoroquinolones actually used in practice.

The susceptibility of therapeutic medications was not determined in $69.6 \%$ of cases analyzed using conventional methods at each facility. The susceptibilities of therapeutic medications were ascertained in $98.9 \%$ of cases with SG17, and the susceptibility results and therapeutic effects were in agreement in $91.3 \%$ of cases. In general, medications are chosen after clinical observation at the first presentation, and practitioners may administer medications evaluated as $\mathrm{R}$ or administer ones with lower therapeutic effects, even though these medications have been evaluated as $\mathrm{S}$ medications. In this study, 13 of 92 patients (14.1\%) required changes to the initial medication. Among these patients, seven were switched to medications with even lower MICs among those evaluated as S. Even when medications evaluated as S were administered from the beginning, information regarding $\mathrm{MIC}$ values may allow the option of switching to even lower MIC drugs, which may help to achieve the desired therapeutic effects more quickly. In contrast, $7.6 \%$ of cases achieved therapeutic effects despite the use of drugs with an R evaluation. This is one of the limitations of an in vitro study.

When one drop of these ophthalmic solutions was instilled, conjunctival tissue concentrations in human beings were $>20 \mu \mathrm{g} / \mathrm{g}$ at 10 minutes after administration. ${ }^{14}$ Fukuda et $\mathrm{al}^{15}$ showed that when two drops of ophthalmic solution were instilled in human eyes every 2 minutes, a total of three times, the corneal tissue concentration at 60 minutes after instillation was $4-13 \mu \mathrm{g} / \mathrm{g}$. Thus, even drugs with an initial $\mathrm{R}$ evaluation may be effective because of their higher concentrations after topical instillation. In our study, we adapted CLSI standards to the results of SG17; however, different standards considering the actual concentrations of drugs in ocular tissues must be established in the future.

As reported previously, ${ }^{7-9}$ it is important to determine drug susceptibility and select the appropriate therapeutics with lower MICs to achieve definitive therapeutic effects during the early phase of treatment. The use of the most efficacious drug will reduce the induction of $\mathrm{R}$ strains. ${ }^{16}$ Therefore, MIC measurements for medications actually used to treat various ophthalmic infections are essential, and SG17 has been shown to be an effective tool for use in this approach.

\section{Conclusion}

SG17 can be used to determine drug susceptibility to antimicrobial agents currently used for treatment in ophthalmic practice. SG17 is more appropriate than conventional methods for selecting antimicrobial drugs.

\section{Acknowledgments}

The abstract of this article was presented at the annual meeting of Association for Research in Vision and Ophthalmology on May 10, 2012, in Fort Lauderdale, FL, USA, as a poster presentation with interim findings. The poster's abstract was published in "Poster Abstracts" in Investigative Ophthalmology \& Visual Science (http://iovs.arvojournals.org/article. aspx? articleid=2359960). ${ }^{17}$

This research was supported by funds from Senju Pharmaceutical Co., Ltd. The funding organization had no role in the design or conduct of this research. We wish to thank Yukari Noguchi from Miyata Eye Hospital, Hiromitsu Fujiwara from Tottori University Hospital, Masahisa Honda from the University of Occupational and Environmental Health, and the late Masako Sakamoto from the Research Foundation for Microbial Diseases of Osaka University for their help with this research.

\section{Disclosure}

The authors report no conflicts of interest in this work.

\section{References}

1. Kowalski RP, Yates KA, Romanowski EG, et al. An ophthalmologist's guide to understanding antibiotic susceptibility and minimum inhibitory concentration data. Ophthalmology. 2005;112(11):1987.

2. Sueke H, Kaye S, Neal T, et al. Minimum inhibitory concentrations of standard and novel antimicrobials for isolates from bacterial keratitis. Invest Ophthalmol Vis Sci. 2010;51(5):2519-2524.

3. Chawla B, Agarwal P, Tandon R, et al. In vitro susceptibility of bacterial keratitis isolates to fourth-generation fluoroquinolones. Eur J Ophthalmol. 2010;20(2):300-305.

4. Sueke H, Kaye SB, Neal T, Hall A, Tuft S, Parry CM. An in vitro investigation of synergy or antagonism between antimicrobial combinations against isolates from bacterial keratitis. Invest Ophthalmol Vis Sci. 2010;51(8):4151-4155.

5. Shalchi Z, Gurbaxani A, Baker M, Nash J. Antibiotic resistance in microbial keratitis: ten-year experience of corneal scrapes in the United Kingdom. Ophthalmology. 2011;118(11):2161-2165.

6. Clinical and Laboratory Standards Institute. Performance Standards for Antimicrobial Susceptibility Testing; Twenty-First Informational Supplement. M100-S21. Wayne, PA: Clinical and Laboratory Standard Institute; 2011. 
7. Lalitha $P$, Srinivasan M, Manikandan $P$, et al. Relationship of in vitro susceptibility to moxifloxacin and in vivo clinical outcome in bacterial keratitis. Clin Infect Dis. 2012;54(10):1381-1387.

8. Kaye S, Tuft S, Neal T, et al. Bacterial susceptibility to topical antimicrobials and clinical outcome in bacterial keratitis. Invest Ophthalmol Vis Sci. 2010;51(1):362-368.

9. Wilhelmus KR, Abshire RL, Schlech BA. Influence of fluoroquinolone susceptibility on the therapeutic response of fluoroquinolone-treated bacterial keratitis. Arch Ophthalmol. 2003;121(9):1229-1233.

10. National Committee for Clinical Laboratory Standards. Methods for Dilution Antimicrobial Susceptibility Tests for Bacteria That Grow Aerobically. Approved Standard M7-A. Villanova, PA: National Committee for Clinical Laboratory Standards; 1986.

11. Clinical and Laboratory Standards Institute. Methods for Dilution Antimicrobial Susceptibility Tests for Bacteria That Grow Aerobically; Approved Standard-Eighth Edition, M7-A8. Wayne, PA: Clinical and Laboratory Standards Institute; 2009.

12. Clinical and Laboratory Standards Institute. Performance Standards for Antimicrobial Disk Susceptibility Tests; Approved Standard - Tenth Edition, A2-A10. Wayne, PA: National Committee for Clinical Laboratory Standards; 2009.
13. Toh N, Nejima R, Ikeda Y, et al. Clinical usefulness of ophthalmic antimicrobial susceptibility plate. Jpn J Clin Ophthalmol. 2011;65(10): 1601-1607.

14. Aihara M, Miyanaga M, Minami K, et al. A comparison of fluoroquinolone penetration into human conjunctival tissue. J Ocul Pharmacol Ther. 2008;24(6):587-591.

15. Fukuda M, Yamada M, Kinoshita S, et al. Comparison of corneal and aqueous humor penetration of moxifloxacin, gatifloxacin and levofloxacin during keratoplasty. Adv Ther. 2012;29(4):339-349.

16. Miyanaga M, Nejima R, Miyai T, et al. Changes in drug susceptibility and the quinolone-resistance determining region of Staphylococcus epidermidis after administration of fluoroquinolones. J Cataract Refract Surg. 2009;35(11):1970-1978.

17. Toh N, Nejima R, Ikeda Y, et al. Clinical utility of Opthalmic Antimicrobial Suscetibility Measurement Plate. Invest Opthalmol Vis Sci. 2012;53:6261.
Clinical Ophthalmology

\section{Publish your work in this journal}

Clinical Ophthalmology is an international, peer-reviewed journa covering all subspecialties within ophthalmology. Key topics include: Optometry; Visual science; Pharmacology and drug therapy in eye diseases; Basic Sciences; Primary and Secondary eye care; Patient Safety and Quality of Care Improvements. This journal is indexed on

Submit your manuscript here: http://www.dovepress.com/clinical-ophthalmology-journal

\section{Dovepress}

PubMed Central and CAS, and is the official journal of The Society of Clinical Ophthalmology (SCO). The manuscript management system is completely online and includes a very quick and fair peer-review system, which is all easy to use. Visit http://www.dovepress.com/ testimonials.php to read real quotes from published authors. 